\title{
Use of NTrap ${ }^{\circledR}$ during Ureteroscopic Lithotripsy for Upper Ureteral Stones
}

\author{
Moung Jin Lee, Seung Tae Lee, Seung Ki Min \\ Department of Urology, National Police Hospital, Seoul, Korea
}

\begin{abstract}
Purpose: This study aimed to determine the value of the NTrap $^{\circledR}$ (Cook Urological INC, USA), which was designed to block the upward movement of stones during ureteroscopic lithotripsy.

Materials and Methods: We reviewed the data of 144 patients who underwent ureteroscopic lithotripsy for an upper ureteral stone from June 2006 to May 2010. Sixty-eight patients who underwent ureteroscopic lithotripsy without the use of the NTrap ${ }^{\mathbb{R}}$ were assigned to Group I and 76 patients who underwent ureteroscopic lithotripsy with the NTrap ${ }^{R}$ were assigned to Group II. The size of the stones, operation time, success rate, and pre- and postoperative complications were compared retrospectively between the two groups.

Results: The mean age of the patients (Group I: 35.8 years; Group II: 32.6 years) and the sex ratios were not significantly different between the two groups. The mean size of the stones was $6.9 \mathrm{~mm}$ and $7.4 \mathrm{~mm}$, which also was not significantly different between the two groups. The mean operation time was 82.7 minutes and 78.7 minutes. The operation time was shorter in Group II, but the difference was not significant. The success rate of stone removal was $89.7 \%$ and $98.7 \%$ in Groups I and II, respectively; Group II showed a significantly higher success rate. Two cases of ureteral perforation and one case of ureteral avulsion occurred in Group I, and one case of ureteral perforation occurred in Group II.

Conclusions: NTrap ${ }^{\mathbb{R}}$, which is an instrument used to assist during ureteroscopic lithotripsy, can be considered to be an effective tool that blocks the upward movement of the stone and aids in safe stone removal.

Key Words: Ureteral calculi; Ureteroscopy

This is an Open Access article distributed under the terms of the Creative Commons Attribution Non-Commercial License (http://creativecommons.org/licenses/by-nc/3.0) which permits unrestricted non-commercial use, distribution, and reproduction in any medium, provided the original work is properly cited.

\author{
Article History: \\ received 17 June, 2010 \\ accepted 23 September, 2010
}

\section{INTRODUCTION}

In the past, there were limitations in treating ureteral calculi, but extensive developments have been made since the introduction of new techniques such as ureteroscopy and extracorporeal shock wave lithotripsy (ESWL). Endoscopic technology equipped with advanced intracorporeal lithotripsy enables the treatment of calculi of the upper urinary tract [1].

Indeed, in addition to small-diameter ureteroscopes, the newly developed techniques of intracorporeal lithotripsy such as ultrasound, electrohydraulic, lithoclast, and more recently the holmium:YAG laser make endoscopic removal of upper ureteral calculi safer and more successful than it had been two decades ago [2-4].

However, stone retropulsion during intracorporeal lithotripsy occurs in $5 \%$ to $40 \%$ of cases. The risk of retropulsion is influenced by the pressure of the irrigant fluid, type of energy source used for intracorporeal lithotripsy, site and degree of calculus impaction, and degree of proximal ureteral dilation [5]. Smaller stones and greater proximal ureteral dilation or hydroureteronephrosis increase the rate of retropulsion.

As solutions for this retropulsion, new instruments such 
as the Accordion ${ }^{\circledR}$ (Percsys, Palo Alto, CA, USA), Stone Cone (Boston Scientific, Natick, MA, USA), and NTrap ${ }^{\mathbb{R}}$ (Cook Urological, Bloomington, IN, USA) have been developed. The NTrap ${ }^{\mathbb{R}}$ is a novel device designed to prevent the retropulsion of ureteral stones and enables safe extraction of stone fragments during ureteroscopic lithotripsy. We present our experience with the use of the NTrap ${ }^{\mathbb{R}}$ during intracorporeal lithotripsy of upper ureteral stones over a 4-year period.

\section{MATERIALS AND METHODS}

In this retrospective study, we reviewed the data of $194 \mathrm{pa}-$ tients who had undergone ureteroscopic stone removal for an upper ureteral stone from June 2006 to May 2010 at our institution. Data from patients who had undergone open surgery and those who had suffered from certain diseases such as active urinary tract infection, congenital anomalies, and previous or access failure were excluded. Ureteroscopic access was successfully achieved for 191 patients (98.5\%). In 47 patients (24.6\%), calculi were retrieved intact by use of a stone basket only, whereas in 144 patients $(75.4 \%)$ lithotripsy was required. Among all subjects, 144 patients $(75.4 \%)$ were identified as having undergone ureteroscopic lithotripsy. For statistical analysis, subjects were divided into Group I and Group II according to the use of the NTrap ${ }^{\circledR}$. Sixty-eight patients who underwent ureteroscopic lithotripsy without the use of the NTrap ${ }^{\circledR}$ were assigned to Group I, and 76 patients who underwent ureteroscopic lithotripsy with the NTrap ${ }^{\circledR}$ were assigned to Group II. Additionally, Group II was divided into two subgroups according to the type of lithotripter used.

The holmium:YAG laser was used in 117 cases (81.3\%). In this observation, the holmium:YAG laser provided successful fragmentation, disintegrating all types of calculi into very small fragments (less than $2 \mathrm{~mm}$ ) that could be easily washed away with irrigation. The Wolf-lithoclast was used for 27 patients (18.8\%), and the fragmentation was successful in all cases. One hundred sixteen $(80.6 \%)$ patients had a single calculus, and the other 28 patients (19.4\%) had more than one calculus. Stone size (maximal diameter on KUB or computed tomography) ranged from 4 to $15 \mathrm{~mm}$.

The size of the stones, operative time, stone-free rate, and intraoperative complications in the two groups were investigated retrospectively. A retrograde ureteroscopic approach was applied for all patients with a 7.5/8 Fr Storz rigid ureteroscope with a $5 \mathrm{Fr}$ working channel. Routine biochemical analysis, blood count, urinalysis, and culture of urine were performed preoperatively. Intravenous pyelogram or computed tomography was taken to confirm the diagnosis and determine the exact locations and sizes of the stones. Prophylactic antibiotics were injected intravenously in all patients.

The operation was performed with the patients under spinal or general anesthesia in the lithotomy position. Introduction of the ureteroscope into the ureter was aided by a 0.038 inch floppy-tipped guidewire. The ureteral orifice was dilated only when necessary for placement of the ureteroscope. In Group II, under fluoroscopic guidance, the NTrap ${ }^{\mathbb{B}}$ was opened above the stone by advancing the inner wire.

The holmium:YAG laser was most frequently used as a lithotripter $(81.3 \%)$. The $550 \mu \mathrm{m}$ fiber was used in all cases. To initiate stone fragmentation, the laser frequency was usually set at a frequency at $6 \mathrm{~Hz}$ and the energy pulse at $0.6 \mathrm{~J}$. The Wolf-lithoclast was the second most commonly used lithotripter (18.7\%) with a $3.5 \mathrm{~mm}$ metal probe. Once the stone was visualized, the lithotriptors were introduced via the ureteroscope and the stone was fragmented. Fragmented stones were removed from the ureter as much as possible by using a basket or forceps. After the operation, every retrieved stone fragment was qualitatively analyzed to verify the chemical composition of each stone. A double $\mathrm{J}$ stent was placed postoperatively in all patients for an average of 1 to 2 weeks.

An operation was regarded as successful when no residual fragments were larger than $2 \mathrm{~mm}$ in size and no additional procedure was required. Postoperative imaging consisted of abdominal plain radiography or computed tomography. A plain radiograph of KUB was performed on the first day after the operation, and after a week, another radiograph was taken to examine residual stone fragments. Most patients were discharged on the first postoperative day. The double $J$ stent was removed after 1 to 2 weeks. All operations were conducted by a single surgeon.

Statistical comparison between Group I and Group II was done by use of mean \pm standard deviation, chi-square test, and Fisher 2 -sided exact test. $\mathrm{p}<0.05$ was considered statistically significant. The SPSS ver. 12.0 (SPSS Inc., Chicago, IL, USA), was used for this statistical analysis.

\section{RESULTS}

The characteristics of the 144 patients are shown in Table 1. No significant differences were observed in age, sex, stone diameter, or type of lithotriptor between Group I and Group II at the time of surgery.

Two weeks after the operations, the success rate was $94.4 \%$. The rates of successful removal of the stones were 89.7\% and $98.7 \%$ for Group I and Group II, respectively. Group II showed a significantly higher success rate than did Group I ( $\mathrm{p}=0.019$ ). For stones $\leq 5 \mathrm{~mm}$ size, the success rate was $92.3 \%$ in Group I and was $100 \%$ in Group II, and no statistically significant difference was observed between the two groups. However, for stones $>5 \mathrm{~mm}$ size, Group II showed a significantly higher rate of successful removal. Group II showed a significantly higher success rate with the use of the holmium:YAG laser as the lithotriptor (Table 2).

Residual stones larger than $2 \mathrm{~mm}$ were noted in 8 cases at 2 weeks after surgery. Among these 8 cases, 6 cases were caused by retropulsion during lithotripsy, and the other resulted from incidental ureteral perforation during litho- 
TABLE 1. Baseline patient characteristics

\begin{tabular}{lccc}
\hline & $\begin{array}{c}\text { Group I } \\
\text { without using the NTrap }\end{array}$ & $\begin{array}{c}\text { Group II } \\
\text { using the NTrap }\end{array}$ & p-value \\
\hline No. of cases & 68 & 76 & 0.618 \\
Men & 50 & 53 & 0.152 \\
Women & 18 & 23 & 0.117 \\
Age (yr) & $35.8 \pm 13.9(18-73)$ & $32.6 \pm 12.3(18-68)$ & \\
Stone diameter (mm) & $6.9 \pm 1.83(4.0-12.0)$ & $7.4 \pm 2.03(4.0-15.0)$ & 0.458 \\
Lithotriptor & 11 & 16 & 60 \\
Wolf-lithoclast & 57 & & \\
Holmium:YAG laser & & & \\
\hline
\end{tabular}

TABLE 2. Success rate, operative time, and intra-operative complications according to the use of the Ntrap

\begin{tabular}{lccc}
\hline & $\begin{array}{c}\text { Group I } \\
\text { without using the NTrap }^{\circledR}\end{array}$ & $\begin{array}{c}\text { Group II } \\
\text { using the NTrap }^{\circledR}\end{array}$ & $p^{\circledR}$-value \\
\hline Overall success rate (\%) & $89.7(61 / 68)$ & $98.7(75 / 76)$ & 0.019 \\
Success rate of stone diameter $\leq 5 \mathrm{~mm}(\%)$ & $92.3(13 / 14)$ & $100(9 / 9)$ & 0.412 \\
Success rate of stone diameter $>5 \mathrm{~mm}(\%)$ & $88.9(48 / 54)$ & $98.5(66 / 67)$ & 0.024 \\
Success rate of Wolf-lithoclast as lithotriptor (\%) & $90.1(10 / 11)$ & $93.8(15 / 16)$ & 0.792 \\
Success rate of holmium:YAG laser as lithotriptor (\%) & $89.5(51 / 57)$ & $100(60 / 60)$ & 0.010 \\
Operative time & $82.6 \pm 14.4(60-120)$ & $78.3 \pm 14.6(50-110)$ & 0.075 \\
Intraoperative complications & 3 & 1 & 0.344 \\
\hline
\end{tabular}

TABLE 3. Failure rate and stone migration rate and treatment of failed patients according to the use of the $\mathrm{Ntrap}^{\mathbb{R}}$

\begin{tabular}{|c|c|c|}
\hline & $\begin{array}{c}\text { Group I } \\
\text { without using } \\
\text { the NTrap }{ }^{\mathbb{R}}\end{array}$ & $\begin{array}{c}\text { Group II } \\
\text { using the } \\
\text { NTrap }^{(}\end{array}$ \\
\hline Failure rate $(\%)$ & $10.3(7 / 68)$ & $1.3(1 / 76)$ \\
\hline Stone migration rate $(\%)$ & $8.8(6 / 68)$ & 0 \\
\hline \multicolumn{3}{|l|}{ Treatment } \\
\hline ESWL & 5 & 1 \\
\hline Repeated URS & 1 & 0 \\
\hline RIRS & 1 & 0 \\
\hline
\end{tabular}

ESWL: extracorporeal shock wave lithotripsy, URS: ureteroscopic removal of stone, RIRS: retrograde intrarenal surgery

tripsy. Failed patients reached a stone-free state with ESWL (6 cases) or repeated ureteroscopic lithotripsy (2 cases; Table 3).

The average durations of the operation in Group I and Group II were 82.6 \pm 14.4 minutes (range, 60-120 minutes) and 78.3 \pm 14.6 minutes (range, 50-110 minutes), respectively. Although Group II showed a shorter mean duration than Group I, the difference was not significant (Table 2). Ureteral avulsion occurred in one patient. The operation stopped immediately to manage the avulsion by open surgery (ureteroureteral anastomosis). The patient was examined with intravenous pyelography after a ureteral stent had been indwelling for 2 months. Ureteral perforations occurred in three male patients who had impacted stones with ureteral narrowing or kinking, because guide wires could not pass the obstructed ureters in these cases.
Intraoperative ureteral perforation was managed by placing an indwelling ureteral stent after stopping the procedure as soon as possible. All patients who suffered from ureteral perforations underwent intravenous pyelography 2 weeks after the removal of the ureteral stent. Ureteral avulsion and perforation, which are types of intraoperative complications, occurred in 4 patients $(2.8 \%)$, and the incidence was $4.4 \%$ in Group I and $1.3 \%$ in Group II, respectively. A higher incidence was noted in Group I, but the difference was not statistically significant ( $\mathrm{p}=0.344$; Table 2 ). There was no evidence of ureteral stricture or extravasation of contrast material in any patient with ureteral avulsion and perforation. In the six cases, postoperative complications including fever were rarely found, two of these six cases had shown urinary tract infection. Three patients had reported postoperative voiding symptoms related to stents. All of them were cleared after stent removal. No long-term complications were found in any cases. There was no significant difference in incidence between the two groups.

\section{DISCUSSION}

As incredible advances in technology have taken place during the past decade, the method of tackling urinary tract stones has been notably refined. These novel therapies such as percutaneous stone treatment, ESWL, and ureteroscopy have virtually replaced open stone surgery. To a certain extent, ESWL is regarded as one of the most preferred treatment modalities for most upper urinary tract stones. Even though ESWL has been verified as a strong therapy, several clinical situations still have to be consid- 
ered as special indications for ureteroscopic removal of calculi. These clinical factors that should be taken into account are previous failure after an ESWL treatment, the existence of firmly impacted (large) stones, and associated distal obstruction [6]. Ureteroscopic stone removal is also a highly recommended option for those patients with bleeding diathesis and those patients for whom ESWL and percutaneous procedures are contraindicated [7].

Recently, ureteroscopy has emerged as the first-line procedure for increasingly challenging stone cases [8]. Kim et al reported that ureteroscopic removal of stones achieved an excellent stone-free rate and a complication rate similar to that of ESWL for treating large $(>10 \mathrm{~mm}$ ) upper ureteral calculi [9]. Furthermore, the miniaturization of ureteroscopes and improved intracorporeal lithotripsy technology has made it possible to successfully access and treat virtually any stone within the ureter in a relatively atraumatic fashion [10].

Proximal calculus migration during ureteroscopy remains a significant problem, however. Stone retropulsion rates for various pneumatic lithotriptors are as high as 15\% to $48 \%[5,11]$. Knispel et al reported $40 \%$ ureteral calculous migration from the proximal ureter and $5 \%$ from the distal ureter [12]. Both pneumatic lithotriptors and holmium:YAG lasers are known to cause calculus retropulsion, the former to a greater extent than the latter. Robert et al reported $48 \%$ calculous migration from the proximal ureter when pneumatic intracorporeal lithotripsy was done [13].

Stone retropulsion increases operative times and cost because it entails a change of the semi-rigid ureteroscope to a flexible instrument in order to chase the migrated stone. Even after the operation, additional procedures might be required to treat residual migrated fragments [6,14].

Stone retropulsion has also been studied in vitro. Marguet et al reported that at clinically relevant settings, the holmium:YAG laser caused less stone retropulsion than did the frequency doubled double pulse Nd:YAG (FREDDY) laser and that both of these lasers caused less retropulsion than the Swiss Lithoclast (EMS, Nyon, Switz- erland) pneumatic lithotriptor [15]. However, the magnitude of stone retropulsion was great enough for all modalities that the authors recommended using an occlusive device such as the Stone Cone during ureteroscopy in all cases.

The occlusive devices represent a new generation of technology that minimize proximal ureteral stone migration. Several new stone retrieval and ureteral occlusive devices have become commercially available over the past few years. Bagley et al have outlined how ureteroscopy has evolved from 1996 to 2004 [16]. Their study showed that, during that time, nitinol baskets supplanted the threeprong grasper for removal of stones and stone fragments. The two novel devices (Stone Cone and NTrap ${ }^{(R)}$ ) have been previously tested and compared for pull-through strength, which is the force required for each device to deploy and release the beads in a strictured ureter model. Ouwenga et al reported statistically significant but clinically insignificant differences in the releasing force of the two devices [17]. The Stone Cone showed a pull-through strength of $0.190 \mathrm{lbf}$ and the $\mathrm{NTrap}^{\circledR}$ device showed a strength of 0.861 lbf, which the group believed was presumably too low to result in ureteral avulsion.

In a recent paper, the Accordion ${ }^{\circledR}$ device was shown to be highly efficient and safe in vitro to prevent stone migration. A clinical trial will have to assess its value in endourological practice [18]. In Korea, Chung et al reported that with the use of the Stone Cone, the success rate of ureteroscopic removal of proximal ureteral stones was $100 \%$ [19].

The NTrap ${ }^{\circledR}$ is a relatively new ureteral occlusive device that prevents the migration of stone fragments during ureteroscopic lithotripsy. The NTrap ${ }^{\mathbb{R}}$ is composed of a tightly woven mesh of nitinol wires that essentially consists of the inner wire and the outer radio-opaque carrying catheter. The inner wire is a shape memory alloy that has a $7 \mathrm{~mm}$ sized umbrella design, namely, the basket (Fig. 1). Its diameter is $2.8 \mathrm{Fr}$ and its total length is $145 \mathrm{~cm}$. Han et al reported the efficacy of the NTrap ${ }^{\mathbb{R}}$ for the treatment of ureteral stones in their initial clinical experience [20]. That
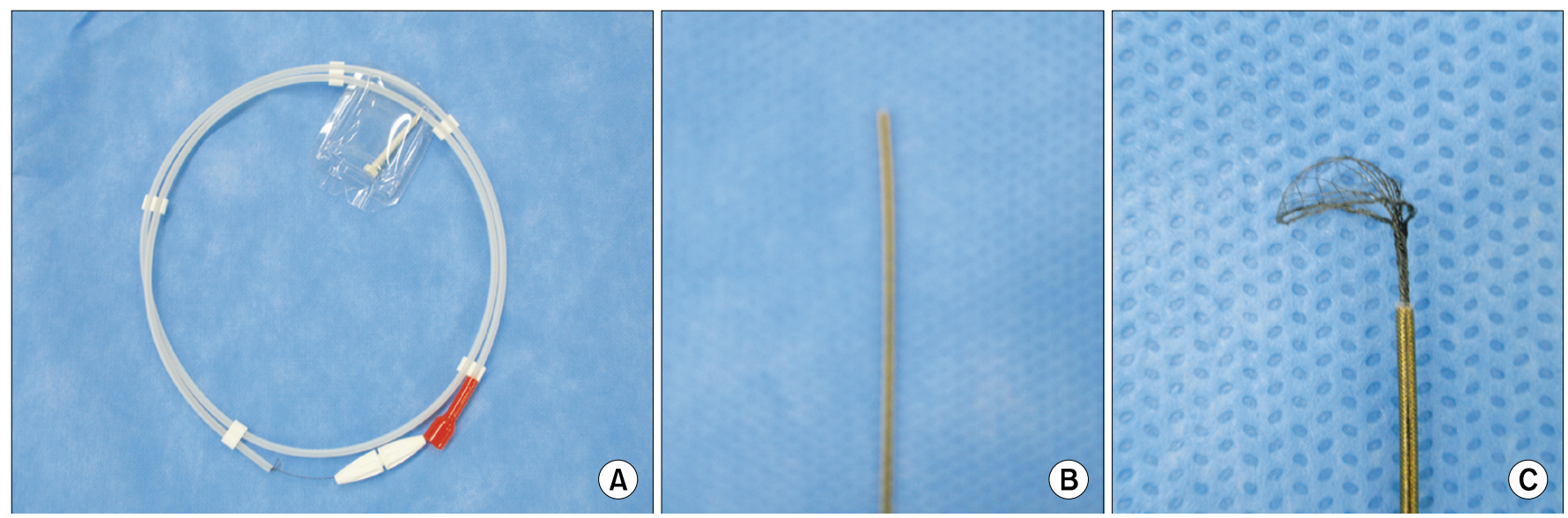

FIG. 1. The NTrap ${ }^{\circledR}$ in its packaging (A), before pushing the inner core (B), and after pushing the inner core (C). 
study revealed that the overall stone-free rate was $100 \%$ and no patient had residual fragments larger than $3 \mathrm{~mm}$ and none required additional treatment. Our study also demonstrated that the NTrap ${ }^{\circledR}$ can increase the success rate of stone removal, especially for stones $>5 \mathrm{~mm}$ size and with the use of the holmium:YAG laser as the lithotriptor. The NTrap ${ }^{\mathbb{R}}$ not only blocks stone retropulsion, but is also effective for removal of residual fragments.

Economic efficiency can be another strong reason for choosing the NTrap ${ }^{\mathbb{R}}[20]$. The Ntrap ${ }^{\mathbb{R}}$ can save time and cost by lowering the rate of stone retropulsion, which involves unnecessary procedures such as prolonged operative times, rigid-flexible ureteroscope alteration, and additional operations.

\section{CONCLUSIONS}

The NTrap ${ }^{\circledR}$ can minimize the risk of stone retropulsion during ureteroscopic lithotripsy. In this study, we found that the use of the NTrap ${ }^{\mathbb{R}}$ can increase the success rate of stone removal, especially for stones $>5 \mathrm{~mm}$ size and with the use of the holmium:YAG laser as the lithotriptor. As a result, the NTrap ${ }^{\mathbb{R}}$, which is an instrument that assists during ureteroscopic lithotripsy, can be an effective tool to prevent stone retropulsion and to aid in safe stone removal.

\section{Conflicts of Interest}

The authors have nothing to disclose.

\section{REFERENCES}

1. Tawfiek ER, Bagley DH. Management of upper urinary tract calculi with ureteroscopic techniques. Urology 1999;53:25-31.

2. Denstedt JD, Clayman RV. Electrohydraulic lithotripsy of renal and ureteral calculi. J Urol 1990;143:13-7.

3. Denstedt JD, Eberwein PM, Singh RR. The Swiss Lithoclast: a new device for intracorporeal lithotripsy. J Urol 1992;148:108890.

4. Scarpa RM, De Lisa A, Porru D, Usai E. Holmium:YAG laser ureterolithotripsy. Eur Urol 1999;35:233-8.

5. Desai MR, Patel SB, Desai MM, Kukreja R, Sabnis RB, Desai RM, et al. The Dretler stone cone: a device to prevent ureteral stone migration-the initial clinical experience. J Urol 2002;167:1985-8.
6. Singal RK, Denstedt JD. Contemporary management of ureteral stones. Urol Clin North Am 1997;24:59-70.

7. Fuchs AMD, Fuchs GJ. Retrograde intra-renal surgery for calculus disease: new minimally invasive treatment approach. $\mathrm{J}$ Endourol 1990;4:337-45.

8. Scales CD, Kang D, Munver R, Auge BK, Ekeruo W, Haleblian GE, et al. Changing practice patterns for the surgical management of renal calculi. J Urol 2006;175(Suppl 4):549.

9. Kim JH, Sung LH, Noh CH. Comparison between rigid ureteroscopic stone removal (URS) and extracorporeal shock wave lithotripsy (ESWL) for large ( $>10 \mathrm{~mm}$ ) upper ureteral stones. Korean J Urol 2006;47:933-7.

10. Zheng W, Denstedt JD. Intracorporeal lithotripsy. Update on technology. Urol Clin North Am 2000;27:301-13.

11. Menezes P, Kumar PV, Timoney AG. A randomized trial comparing lithoclast with an electrokinetic lithotripter in the management of ureteric stones. BJU Int 2000;85:22-5.

12. Knispel HH, Klän R, Heicappell R, Miller K. Pneumatic lithotripsy applied through deflected working channel of miniureteroscope: results in 143 patients. J Endourol 1998;12:513-5.

13. Robert M, Bennani A, Guiter J, Avérous M, Grasset D. Treatment of 150 ureteric calculi with the Lithoclast. Eur Urol 1994;26: 212-5.

14. Sun Y, Wang L, Liao G, Xu C, Gao X, Yang Q, et al. Pneumatic lithotripsy versus laser lithotripsy in the endoscopic treatment of ureteral calculi. J Endourol 2001;15:587-90.

15. Marguet CG, Sung JC, Springhart WP, L'Esperance JO, Zhou S, Zhong $\mathrm{P}$, et al. In vitro comparison of stone retropulsion and fragmentation of the frequency doubled, double pulse nd:yag laser and the holmium:yag laser. J Urol 2005;173:1797-800.

16. Bagley DH, Slotoroff CB, Zeltser IS. Evolution of ureteroscopy from 1996 to 2004: comparison of indications, endoscopes and instruments. J Endourol 2005;19(Suppl 1):75.

17. Ouwenga MK, Sharma SK, Holley P, Turk TM, Perry KT. Load-release points of two novel ureteral stone-trapping devices. J Endourol 2005;19:894-7.

18. Olbert PJ, Keil C, Weber J, Schrader AJ, Hegele A, Hofmann R. Efficacy and safety of the Accordion stone-trapping device: in vitro results from an artificial ureterolithotripsy model. Urol Res 2010;38:41-6.

19. Chung HS, Park JY, Kim HK, Park CM. Efficacy of the Stone Cone for treatment of proximal ureteral stones: an initial clinical experience. Korean J Urol 2006;47:412-7.

20. Han DJ, Moon HY, Kim CS. Efficacy of the NTrap ${ }^{\circledR}$ for the treatment of ureteral stone: an initial clinical experience. Korean $\mathrm{J}$ Urol 2007;48:1161-4. 